\title{
A NOTE ON EXACT PARTICULAR SOLUTIONS OF THE GENERALIZED SHALLOW-WATER EQUATIONS
}

BY

\author{
ANATOLY B. ODULO
}

Applied Science Associates, Inc., Narragansett, RI

Abstract. This note presents a set of systems of two first-order quasi-linear partial differential equations, which can be reduced to the shallow-water equations. This set includes equations describing a two-layered fluid flow.

Introduction. The shallow-water equations

$$
u_{t}+u u_{x}+h_{x}=0, \quad h_{t}+u h_{x}+h u_{x}=0
$$

have been intensively studied analytically and numerically (see, e.g., [2], [4, §13.10]). Several exact analytical solutions of (SW) are known:

$$
\begin{aligned}
& u=x / t, \quad h=a / t ; \quad u=(b+2 x / t) / 3, \quad h=(x / t-b)^{2} / 9+a / t^{2 / 3} ; \\
& u=f^{\prime}(t) x / f(t), \quad 4 h=a\left(1-x^{2} / f^{2}(t)\right) / f(t), \quad f^{\prime}(t)=(1-a / f(t))^{1 / 2} \\
& x=2 h t^{2} s+0.5 \ln (1+2 s /(1-s)), \quad u=2 h t s, \quad s^{2}=1-h /\left(b-h^{2} t^{2}\right) ;
\end{aligned}
$$

Here $a$ and $b$ are arbitrary constants, and ' denotes the derivative. Consider the generalized shallow-water equations:

$$
v_{t}+F(v, \zeta) v_{x}+0.5 G_{1}(v, \zeta) \zeta_{x}=0, \quad \zeta_{t}+F(v, \zeta) \zeta_{x}+0.5 G_{2}(v, \zeta) v_{x}=0 .
$$

ThEOREM. Let $u=u(x, t)$ and $h=h(x, t)$ be a solution of (SW).

a) If

$$
F(v, \zeta)=f_{1}(v+\zeta)+f_{2}(v-\zeta) \quad \text { and } \quad G_{1}(v, \zeta)=G_{2}(v, \zeta)=f_{1}(v+\zeta)-f_{2}(v-\zeta)
$$

then relations

$$
f_{1}(v+\zeta)+f_{2}(v-\zeta)=u(x, t) \quad \text { and } \quad f_{1}(v+\zeta)-f_{2}(v-\zeta)=2 h^{1 / 2}(x, t)
$$

give a solution of (GSW).

Received March 17, 1999.

2000 Mathematics Subject Classification. Primary 35Q35, 35F20, 76B70. 
b) If

$$
F(v, \zeta)=f_{1}(v) f_{2}(\zeta)+q, \quad G_{1}(v, \zeta)=\left(c_{1}+f_{1}^{2}\right) f_{2}^{\prime} / f_{1}^{\prime},
$$

and

$$
G_{2}(v, \zeta)=\left(c_{2}+f_{2}^{2}\right) f_{1}^{\prime} / f_{2}^{\prime}
$$

then relations

$$
f_{1}(v) f_{2}(\zeta)+q=u(x, t) \text { and }\left(c_{1}+f_{1}^{2}\right)\left(c_{2}+f_{2}^{2}\right)=4 h(x, t)
$$

give a solution of (GSW). Here $f_{1}$ and $f_{2}$ are arbitrary functions, and $q$ is a constant.

Proof. Inserting $u=F(v, \zeta)$ and $h=G(v, \zeta)$ into (SW), we get

$$
v_{t}+(F+A(v, \zeta)) v_{x}+B_{1}(v, \zeta) \zeta_{x}=0, \quad \zeta_{t}+(F-A(v, \zeta)) \zeta_{x}+B_{2}(v, \zeta) v_{x}=0 .
$$

Here $A=\left(G_{v} G_{\zeta}-G F_{v} F_{\zeta}\right) / D, B_{1}=\left(G_{\zeta}^{2}-G F_{\zeta}^{2}\right) / D, B_{2}=\left(G F_{v}^{2}-G_{v}^{2}\right) / D$, and $D(v, \zeta)=$ $F_{v} G_{\zeta}-G_{v} F_{\zeta}$

a) Considering $F(v, \zeta)=f_{1}(v+\zeta)+f_{2}(v-\zeta)$ and $G=\left[f_{1}(v+\zeta)-f_{2}(v-\zeta)\right]^{2} / 4$ we obtain $A=0, B_{1}=B_{2}=\left[f_{1}(v+\zeta)-f_{2}(v-\zeta)\right] / 2$. Thus (5) becomes (GSW) with $F, G_{1}$, and $G_{2}$ as given by (1).

b) Considering $F(v, \zeta)=f_{1}(v) f_{2}(\zeta)+q$ and $G=\left(c_{1}+f_{1}^{2}\right)\left(c_{2}+f_{2}^{2}\right) / 4$ we obtain $A=0$, $B_{1}=0.5\left(c_{1}+f_{1}^{2}\right) f_{2}^{\prime} / f_{1}^{\prime}, B_{2}=0.5\left(c_{2}+f_{2}^{2}\right) f_{1}^{\prime} / f_{2}^{\prime}$. Thus (5) becomes (GSW) with $F, G_{1}$, and $G_{2}$ as given by (3).

EXAMPLE 1. Taking $f_{1}(z)=f_{2}(z)=z^{n}$ in (1) we get (GSW) with $F=(v+\zeta)^{n}+$ $(v-\zeta)^{n}, G_{1}=G_{2}=(v+\zeta)^{n}-(v-\zeta)^{n}$. This equation has exact analytical solutions $2 v(x, t)=\left(u / 2+h^{1 / 2}\right)^{1 / n}+\left(u / 2-h^{1 / 2}\right)^{1 / n}, 2 \zeta(x, t)=\left(u / 2+h^{1 / 2}\right)^{1 / n}-\left(u / 2-h^{1 / 2}\right)^{1 / n}$ with $u(x, t)$ and $h(x, t)$ from (PSa-d).

ExAmple 2 (Unsteady two-layer fluid flow). Taking $f_{1}(x)=f_{2}(x)=x$ and $c_{1}=$ $c_{2}=-1$ in (3) we get (GSW) in the form

$$
v_{t}+(v \zeta+q) v_{x}-0.5\left(1-v^{2}\right) \zeta_{x}=0, \quad \zeta_{t}+(v \zeta+q) \zeta_{x}-0.5\left(1-\zeta^{2}\right) v_{x}=0 .
$$

This system describes a flow of two homogeneous inviscid fluids between two horizontal rigid plates in the hydrostatic and Boussinesq approximations (see (3.3.12) in [1]). The velocity $u_{1}, u_{2}$ and the thickness $h_{1}, h_{2}$ of the upper lighter (density $\rho_{1}$ ) and lower denser (density $\rho_{2}$ ) layer (see Fig. 1) are expressed in terms of $v$ and $\zeta$ as follows:

$$
\begin{aligned}
& u_{1} / c_{0}=q+v(1+\zeta) / 2, \quad u_{2} / c_{0}=q-v(1-\zeta) / 2, \\
& h_{1} / H=(1-\zeta) / 2, \quad h_{2} / H=(1+\zeta) / 2
\end{aligned}
$$

Here $H$ is a distance between the plates, $c_{0}^{2}=g^{\prime} H, g^{\prime}=g\left(\rho_{2}-\rho_{1}\right) / \rho_{2}, g$ is the acceleration of gravity, $q=Q / c_{0} H, Q$ is the total volume flux, and $-1<\zeta<1$.

If $u(x, t), h(x, t)$ is a solution of (SW), then $v=w_{+}+w_{-}, \zeta=w_{+}-w_{-}$is a solution of (SW2). Here $w_{ \pm}=\left[(1 \pm u(x-q t, t))^{2} / 4-h(x-q t, t)\right]^{1 / 2}$. Using (PSa-d) we get exact analytical solutions of (SW2). In particular, using (PSd) with $b=1 / 4$ we have

$$
\begin{aligned}
x-q t & =\zeta\left(1-\zeta^{2}\right) t^{2} /\left[1+\left(1+\left(1-\zeta^{2}\right)^{2} t^{2}\right)^{1 / 2}\right]+0.5 \ln (1+2 \zeta /(1-\zeta)), \\
v & =\left(1-\zeta^{2}\right) t /\left[1+\left(1+\left(1-\zeta^{2}\right)^{2} t^{2}\right)^{1 / 2}\right] .
\end{aligned}
$$




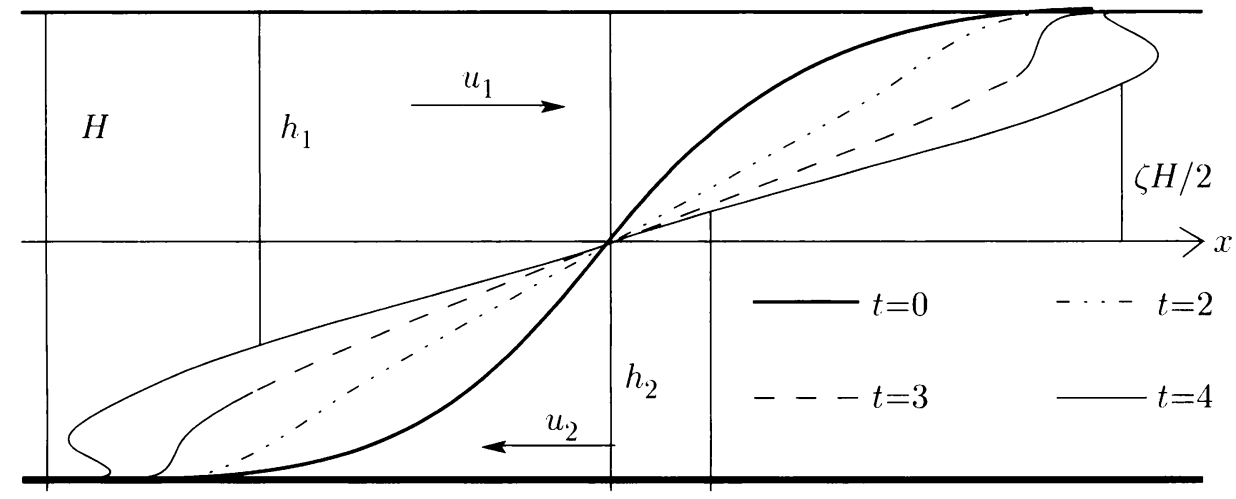

FIc. 1. Evolution of the interface described by (SW2) with $q=0$ for initial conditions $v(x, 0)=0, \zeta(x, 0)=\tanh x$

This is the solution of (SW2) with initial conditions $\zeta(x, 0)=\tanh x, v(x, 0)=0$. Let $q=0$. For $x \ll 1$ we have $\zeta \approx x\left(1+t^{2}\right)^{1 / 2}$ and $v \approx t /\left[1+\left(1+t^{2}\right)^{1 / 2}\right]$. The maximum of $v(x, t)$ is at $x=0$ and increases from 0 to 1 when $t$ increases from 0 to infinity. Figure 1 shows the position of the interface for $t=0,2,3$, and 4 .

\section{REFERENCES}

[1] P. G. Baines, Topographic effects in stratified flows, Cambridge University Press, New York, 1995

[2] J. Gratton and C. Vigo, Self-similar gravity currents with variable inflow revisited: Plane currents, J. Fluid Mech. 258, 77104 (1994)

[3] A. B. Odulo, A method of obtaining exact particular solutions of a system of two first-order quasilinear partial differential equations, Quart. J. Mech. Appl. Math. 44(2), 323-331 (1992)

[4] G. B. Whitham, Linear and nonlinear waves, Wiley-Interscience, New York, 1974 